\title{
Conductive polymer foams with carbon nanofillers - Modeling percolation behavior
}

\author{
O. Maxian, D. Pedrazzoli, I. Manas-Zloczower ${ }^{*}$ \\ Department of Macromolecular Science and Engineering, Case Western Reserve University, 2100 Adelbert Road, \\ 44106-7202 Cleveland (OH), USA
}

Received 28 October 2016; accepted in revised form 11 January 2017

\begin{abstract}
A new numerical model considering nanofiller random distribution in a porous polymeric matrix was developed to predict electrical percolation behavior in systems incorporating 1D-carbon nanotubes (CNTs) and/or 2D-graphene nanoplatelets (GNPs). The numerical model applies to porous systems with closed-cell morphology. The percolation threshold was found to decrease with increasing porosity due to filler repositioning as a result of foaming. CNTs were more efficient in forming a percolative network than GNPs. High-aspect ratio (AR) and randomly oriented fillers were more prone to form a network. Reduced percolation values were determined for misaligned fillers as they connect better in a network compared to aligned ones. Hybrid CNT-GNP fillers show synergistic effects in forming electrically conductive networks by comparison with single fillers.
\end{abstract}

Keywords: modeling and simulation, carbon nanotubes, graphene, hybrid systems, porous materials

\section{Introduction}

Polymer foams exhibit attractive features such as low density, elevated specific impact and damping properties, and high thermal insulation, making them suitable for a variety of applications, including thermal and acoustic insulation, medical devices, construction, transportation, and protective equipment [1]. Considerably light polymer foams, characterized by densities as low as $0.05 \mathrm{~g} / \mathrm{cm}^{3}$, can be successfully fabricated using blowing agents such as supercritical $\mathrm{CO}_{2}$ [2-4]. Potential applications of such foams in the field of electrically and thermally conductive materials, as well as electromagnetic interference (EMI) shielding materials can be developed by incorporating conductive nanofillers such as carbon nanotubes (CNTs) and graphene nanoplatelets (GNPs) within the porous matrix prior to foaming [5]. Owing to their high aspect ratio (AR) and exceptional mechanical and electrical properties, CNTs and GNPs offer important advantages over conventional nanofillers. For instance, they can simultaneously enhance the mechanical properties and electrical conductivity at very low filler content (i.e. $<0.1 \mathrm{wt} \%$ ) [6-9]. Xu et al. [2] described the fabrication of conductive polyurethane composites containing CNTs with density of around $0.05 \mathrm{~g} / \mathrm{cm}^{3}$, observing a density-dependent conductor-insulator transition which defines the limit of weight reduction for a conductive composite foam. Furthermore, the conductor-insulator transition was related to the microstructural features of the composite foam.

However, modeling and optimization of conductive polymer foams have only been scarcely reported [10]. Behbahani et al. [11] reported the effect of foaming on the percolation behavior of polymer composites incorporating carbon fibers and CNTs. Although they observed good agreement with experimental data for non-porous systems, their simulation results

${ }^{*}$ Corresponding author, e-mail: $\underline{\text { ixm@,case.edu }}$ (C) BME-PT 
showed no significant dependency of the percolation threshold on the porosity level. This was in contrast to experimental results, which showed an increase in electrical conductivity and a decrease in percolation threshold with increasing porosity when considering the CNT volume fraction with respect to the composite total volume. The authors attributed this discrepancy to the effect of foaming on the filler network morphology, as foaming may induce filler particle rearrangement, thus resulting in a more efficient network.

Thus there is a need to further investigate and model the relationships between foam morphology (porosity level, cell size distribution, cell type and shape) and nanofiller loadings required to achieve electrical conductivity in porous nanocomposites. The aim of the current study is to model the electrical percolation behavior of polymer foams incorporating 1D- and 2D-nanofillers by accurately capturing changes in system morphology that occur upon foaming. In a previous work carried out by our group, we proposed a new $\mathrm{C}++$ numerical model to investigate $3 \mathrm{D}$-electrical percolation behavior of non-porous polymer nanocomposites incorporating CNT and GNP fillers, assuming a random filler distribution and orientation and perfect filler-matrix interfacial bonding [12]. Here we extend this model to study percolation behavior in polymer foams. In particular, the model uses (i) random sequential addition (RSA) of fillers in the matrix prior to incorporating the pores and at concentration levels below percolation in solids and (ii) RSA of spherical pores with subsequent filler relocation to the pore edges for those fillers that would land inside the pores. The formation of a continuous filler network (percolation behavior) is evaluated at different levels of porosity. With this novel procedure, the model is able to capture the real experimental methodology in making foams in micro-cellular injection molding.

\section{Simulation procedure}

\subsection{Electrical percolation in porous nanocomposites}

When considering foaming of a polymer nanocomposite, fillers are mixed within a polymer matrix containing small droplets of a supercritical fluid, such as supercritical $\mathrm{CO}_{2}$. Pressure of the system is then increased and the fluid evaporates, leaving 'pores' (cavities or empty spaces where there are no fillers). Fillers originally residing inside the fluid droplets are pushed out to the pore edges, where they can interact with fillers already in the matrix thus facilitating network formation. Our simulations begin with a system with fillers already inside the matrix, at a volume fraction lower than the percolation threshold typical for the non-porous system (initially $\sim 10 \%$ lower). Subsequently, pores are progressively added to the system in a random position one at a time, with fillers inside the pores being repositioned until a filler network ( $80 \%$ of the fillers being in contact with two or more other fillers) is formed. System porosity is recorded, and the percolation threshold is reported as solid volume fraction, as shown by Equation (1):

$$
\begin{aligned}
& \text { Percolation volume fraction }[\%]= \\
& =\frac{\text { volume of fillers }}{\text { total volume }- \text { volume of pores }} \cdot 100
\end{aligned}
$$

In this study, pores are modeled as spheres with diameters within a normal distribution centered on some mean value and a standard deviation of $10 \%$ of the mean. The mean pore diameter was selected to be small relative to the representative volume element (RVE) used in the simulations (so that many pores could fit inside the RVE), but large with respect to the filler dimension (so that a number of fillers could fit completely inside a pore).

\subsection{Electrical percolation in polymer/CNT systems}

Consider two CNTs, $\mathrm{CNT}_{1}$ and $\mathrm{CNT}_{2}$, with coordinates of the two ends represented by $\left(x_{1}, y_{1}, z_{1}\right),\left(x_{2}\right.$, $\left.y_{2}, z_{2}\right)$ and $\left(x_{3}, y_{3}, z_{3}\right),\left(x_{4}, y_{4}, z_{4}\right)$, respectively and directions described by the vectors $\mathbf{d}_{1}$ and $\mathbf{d}_{2}$, respectively as shown by Equations (2a) and (2b):

$$
\begin{aligned}
& \mathbf{d}_{1}=\left(x_{2}-x_{1}, y_{2}-y_{1}, z_{2}-z_{1}\right) \\
& \mathbf{d}_{2}=\left(x_{4}-x_{3}, y_{4}-y_{3}, z_{4}-z_{3}\right)
\end{aligned}
$$

Thus, the two specific fillers/tubes can be formally represented as Equation (3):

$$
\begin{aligned}
& \mathrm{CNT}_{1}=s_{1}+\mathbf{d}_{1} \cdot s \\
& \mathrm{CNT}_{2}=s_{2}+\mathbf{d}_{2} \cdot t
\end{aligned}
$$

where $s_{\mathrm{i}}$ is the start location of filler $i, \mathbf{d}_{\mathrm{i}}$ is defined as in Equation (2), and $s$ and $t$ are scalar values between 0 and 1, inclusive. The distance between these two fillers is: 


$$
\left\|\mathrm{CNT}_{1}-\mathrm{CNT}_{2}\right\|=\left\|s_{1}-s_{2}+\mathbf{d}_{1} \cdot s-\mathbf{d}_{2} \cdot t\right\|
$$

To determine the minimum distance between the two CNTs, Equation (4) is minimized with respect to $s$ and $t$, such that $s_{\min }$ and $t_{\min }$ fall within the domain $[0,1]$. The minimum of the function is then evaluated, and two tubes are considered in contact (Figure 1a) when the distance is smaller than $\left(d_{\text {cut-off }}+\right.$ tube diameter), where $d_{\text {cut-off }}$ is the cut-off distance for electron tunneling, set according to the current literature on electrical conductivity in polymer nanocomposites (between 1 and $10 \mathrm{~nm}$ ) [13, 14]. In this work CNTs of length $1.125 \mu \mathrm{m}$ and diameter $9.75 \mathrm{~nm}$ (dimensions chosen based on representative experimental data [15]) were used to test the model in bulk solids using a cutoff distance of $7.5 \mathrm{~nm}$, which is within the range reported by Ambrosetti et al. [16] for CNTs with similar AR. The simulations used an RVE or cuboid with a lateral edge length of $10 \mu \mathrm{m}$ and a single cuboid boundary condition. Cuboid dimensions were chosen such that the cuboid edge was large compared to the larger filler dimension, which allowed for the use of the single cuboid boundary condition, while not being prohibitively large as to result in unwieldy computational times.

These simulations for the electrical percolation threshold in non-porous systems rendered results in line with reported experimental values in the range of $0.0025-2 \mathrm{vol} \%$ for different thermoplastic and thermoset matrices [16-17].

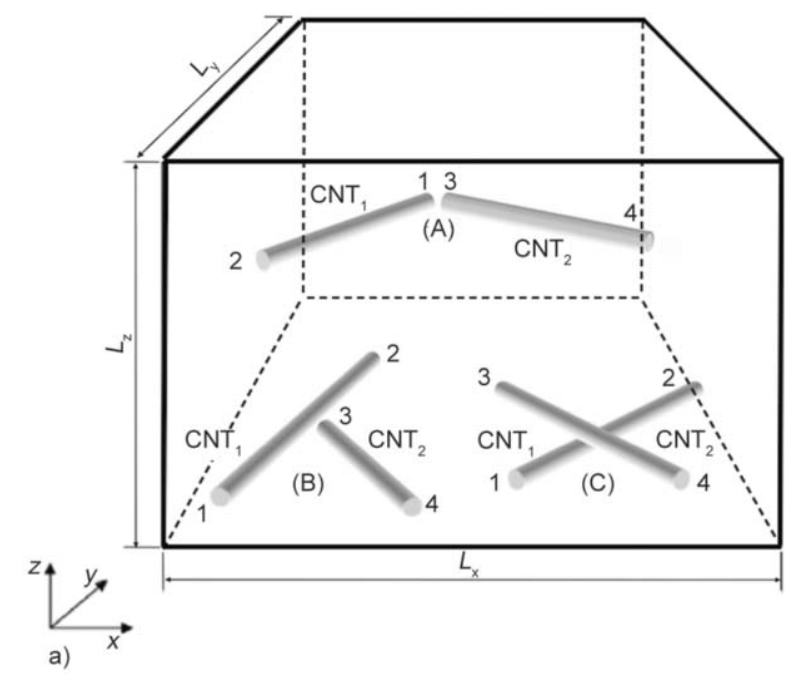

\section{Electrical percolation in porous $C N T$ nanocomposites}

In order to calculate the CNT percolation threshold in porous systems, filler location with respect to the pores must first be determined. To do this, we consider the center of a sphere/pore projected onto the infinite line that contains the CNT to determine the point on the CNT closest to the center of the sphere. As shown in the two-dimensional schematic (Figure $1 \mathrm{~b}$ ), the closest point from the pore center on the CNT can be found by Equation (5):

$p_{c}=s+(\mathbf{p} \cdot \mathbf{c}) \mathbf{c}$

where $p_{\mathrm{c}}$ is the closest point from the sphere center on the CNT, $s$ is the start location of the CNT, $\mathbf{p}$ is a vector from the pore center to the start location, and c is a unit vector that describes the CNT (i.e. = $\left(\mathrm{CNT}_{\text {end }- \text { start }} /\right.$ /ength $)$, as described in Figure 1. Note that if $(\mathbf{p} \cdot \mathbf{c})<0, p_{\mathrm{c}}=\mathrm{CNT}$ start location, and if (p.c) $>$ CNT length, $p_{\mathrm{c}}=\mathrm{CNT}$ end location.

We define $\mathbf{r}$ as the vector from the closest point on the CNT to the pore center. If $\|\mathbf{r}\|<R$, where $R$ is the pore radius, then the CNT is inside the pore. Otherwise it is not, and the CNT is not modified (this is the case represented in Figure 1b). If the CNT is inside the pore, then it is moved to the pore edge along the vector $r$ given by Equation (6):

$P_{2}=P_{1}+\left(\frac{R}{\|\mathbf{r}\|}-1\right) \mathbf{r}$

Figure 1. (a) Contact patterns between pairs of CNTs. CNTs can contact via their ends, lateral edges, or between a lateral edge and an end. (b) Finding $p_{\mathrm{c}}$ (the closest point on the filler to the pore center) as the projection of the pore center onto the line containing the CNT, with $p_{\mathrm{c}}$ being confined to the CNT start or endpoint if the projection falls outside the CNT. 

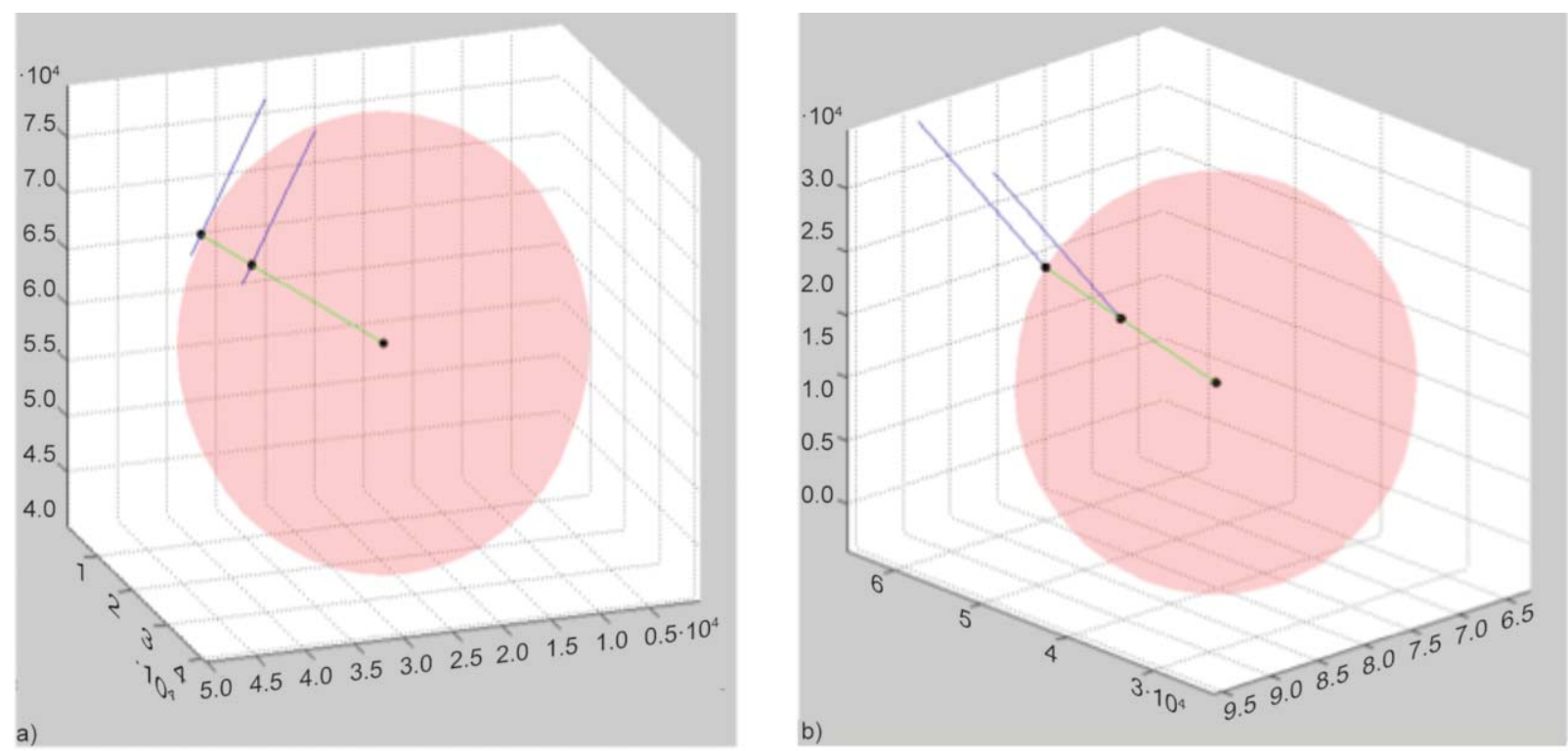

Figure 2. Moving a CNT out of a pore in two cases: (a) the point on the CNT closest to the pore center $\left(p_{\mathrm{c}}\right)$ is on the body of the CNT; (b) $p_{\mathrm{c}}$ is an endpoint of the CNT. The CNT is moved out of the pore along the vector $\mathbf{r}$ containing the pore center and $p_{\mathrm{c}}$ shown in green.

where $P_{1}$ denotes any point on the original CNT and $P_{2}$ the same point on the CNT now on the edge of the pore. Note that by construction if $\|\mathbf{r}\|=R$, the CNT is on the edge of the pore and no motion occurs. Figure 2 demonstrates how a CNT is moved out of a pore.

\subsection{Electrical percolation in polymer/GNP systems}

A single circular GNP is defined by a center, radius, and set of three orthonormal vectors, $\mathbf{u}, \mathbf{v}, \mathbf{n}$, where $\mathbf{u}$ and $\mathbf{v}$ are the vectors that form a basis for the plane of

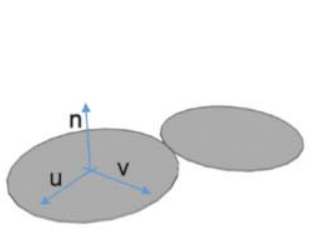

(A)

a) the disk and $\mathbf{n}$ is the vector normal to the plane of the disk (Figure 3a). As defined in [18], contacts between two disks, $\mathrm{GNP}_{1}$ and $\mathrm{GNP}_{2}$, can be determined by minimizing the distance function between them. Let $P_{1}$ and $P_{2}$ be the points on $\mathrm{GNP}_{1}$ and $\mathrm{GNP}_{2}$ that minimize the distance between them. There are four possible contact patterns: (A) both points lie on the circumference of their respective disks; (B) and (C) either $P_{1}$ or $P_{2}$ lies on the interior of its repective disk and the other point lies on the circumference and (D) $P_{1}$ and $P_{2}$ lie on the interior of their respective disks (Figure 3a). Case (D) corresponds to an intersection

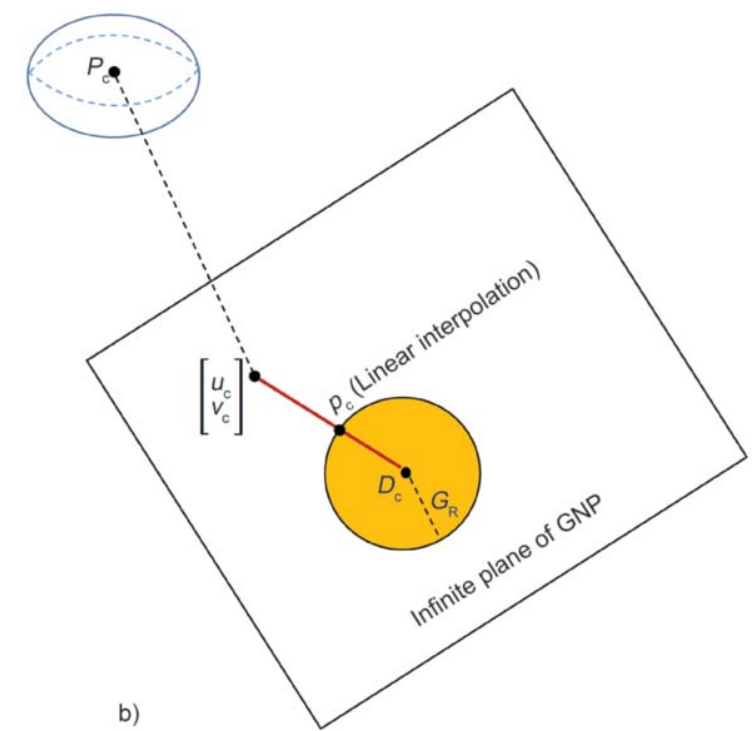

Figure 3. (a) Contact patterns between pairs of GNPs. GNPs can contact via their edges or between an edge and an interior. (b) Procedure for determining the closest point on a GNP to a pore center, $p_{\mathrm{c}}$. The pore center is projected onto the infinite plane of the GNP, and if this point is not on the GNP, $p_{\mathrm{c}}$ is found via interpolation as shown. 
between the two disks (or in this algorithm the case that a line orthogonal to both normal vectors contains at least one point that is on $\mathrm{GNP}_{1}$ and $\mathrm{GNP}_{2}$ ), in which case $\mathrm{GNP}_{2}$ is not added to the cuboid [12]. Case (A) has no closed-form solutions. To address this, we follow the iterative algorithm proposed by Almohamad [18]. In cases (B) and (C), Almohamad obtained closed-form solutions on the infinite plane of the disk, and here we check that those solutions actually do fall on the GNP. For more details on the procedures of contacting GNPs, see [12].

The model was first tested on non-porous systems with GNPs of diameter $0.75 \mu \mathrm{m}$ and thickness $11.25 \mathrm{~nm}$ (corresponding to experimentally reported data) [19] using a cuboid with lateral edge length of $10 \mu \mathrm{m}$ and a cutoff distance of $7.5 \mathrm{~nm}$ (in the range reported by Ambrosetti for GNPs of similar AR [16]). The obtained percolation values of $8-10 \mathrm{vol} \%$ were within the experimental range for GNPs with similar AR $[14,20]$.

\section{Electrical percolation in porous GNP nanocomposites}

In order to determine the percolation threshold for porous polymer/GNP systems, filler location with respect to the pores must first be determined. To do this, we begin by calculating the coordinates of the pore center with respect to the disk basis, as shown by Equation (7):

$\left[\begin{array}{l}u_{\mathrm{c}} \\ v_{\mathrm{c}} \\ n_{\mathrm{c}}\end{array}\right]=\left[\begin{array}{l}-\mathbf{u}- \\ -\mathbf{v}- \\ -\mathbf{n}-\end{array}\right]\left(P_{\mathrm{c}}-D_{\mathrm{c}}\right)$

where $P_{\mathrm{c}}$ and $D_{\mathrm{c}}$ denote the pore and disk center coordinates with respect to the standard basis. Now, if $\left|n_{\mathrm{c}}\right|$ is greater than the radius of the pore, then the entire GNP (where $n=0$ ) is at least a pore radius away from the pore center and there is no need to continue. If $\left|n_{\mathrm{c}}\right|$ is less than the radius of the pore, it is possible for the GNP to intersect the pore. The next task is to find the point on the GNP that is closest to the pore center. If according to Equation (8):

$\sqrt{u_{\mathrm{c}}^{2}+v_{\mathrm{c}}^{2}} \leq G_{\mathrm{R}}$

where $G_{\mathrm{R}}$ is the radius of the GNP, then the projection of the pore center falls on the GNP, and this point is the closest point on the GNP to the pore center, $p_{\mathrm{c}}$. Otherwise, a linear interpolation is performed. The closest point on the GNP to the pore center is the point on the GNP that is closest to the projection of the pore center onto the infinite plane that contains the GNP, as shown in Figure 3b. Mathematically, since the projection of the pore center onto the plane
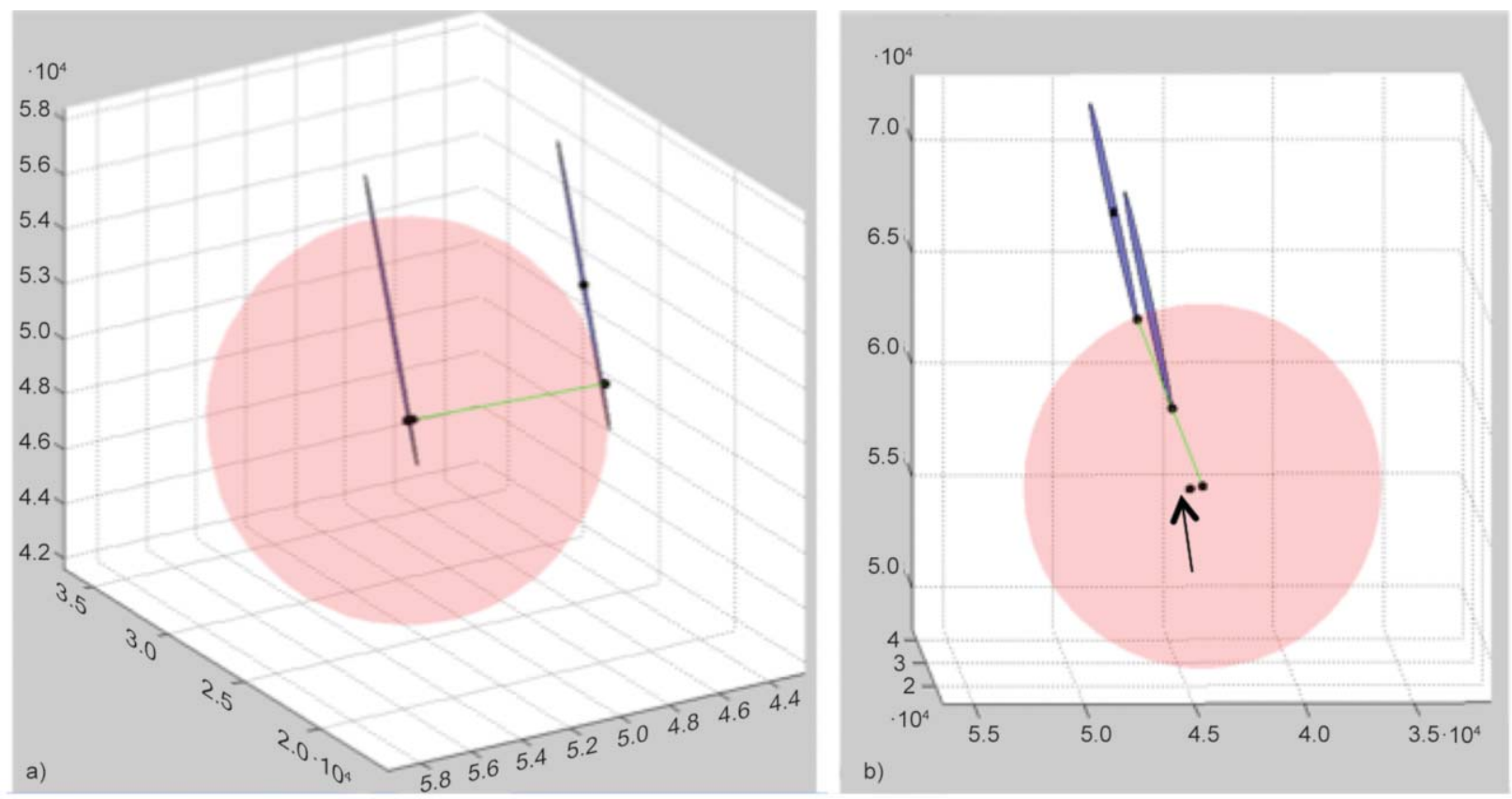

Figure 4. Moving a GNP out of a pore in two cases: (a) the projection of the pore center is on the GNP and is therefore $p_{\mathrm{c}}$, (b) the projection of the pore center (black arrow) is not on the GNP and $p_{\mathrm{c}}$ is found via linear interpolation. In both cases, the GNP is moved out of the pore along the line from the pore center to $p_{\mathrm{c}}$. 
of the GNP can be represented by the vector $\left[\begin{array}{l}u_{\mathrm{c}} \\ v_{\mathrm{c}}\end{array}\right]$, the
point $p_{\mathrm{c}}$ can be found by Equation (9):

$p_{\mathrm{c}}=\left[\begin{array}{c}u_{\mathrm{c}} \\ v_{\mathrm{c}}\end{array}\right] \frac{G_{\mathrm{R}}}{\sqrt{u_{\mathrm{c}}^{2}+v_{\mathrm{c}}^{2}}} \quad \sqrt{u_{\mathrm{c}}^{2}+v_{\mathrm{c}}^{2}}>G_{\mathrm{R}}$

$p_{\mathrm{c}}=\left[\begin{array}{c}u_{\mathrm{c}} \\ v_{\mathrm{c}}\end{array}\right] \quad \sqrt{u_{\mathrm{c}}^{2}+v_{\mathrm{c}}^{2}} \leq G_{\mathrm{R}}$

Once $p_{\mathrm{c}}$ is identified, its coordinates in the standard basis are found and it is determined whether or not $p_{\mathrm{c}}$ is inside the pore. If it is, then Equation (6) is used to move the GNP outward radially along the line containing the pore center and $p_{\mathrm{c}}$ until the entire GNP is outside the pore. Figure 4 demonstrates how the GNP is moved out of the pore. Unlike CNT systems, where filler intersections are negligible, GNPs could contact or intersect neighboring GNPs once they are moved out of the pore. In this study, these intersections are treated as contacts within the percolating network in order to simplify the model.

Secondary effects typically occurring during foaming of polymer nanocomposites, such as filler accumulation on the cell walls or in the struts were not taken into account when moving fillers out of the pores due to the complexity of modeling such features.

\subsection{Electrical percolation in hybrid systems}

Hybrid systems with CNT/GNP relative volume ratios 30/70, 50/50 and 70/30 were examined in this work. We have applied a new closed-form method to determine whether or not a CNT is in contact with a
GNP. Specifically, the algorithm determines what section of the CNT could be in contact with the GNP in the normal direction, then minimizes the planar distance to determine whether or not some part of the CNT is close enough to be considered in contact with the GNP.

It is known from the literature that fillers of different size and geometry such as CNTs and GNPs can potentially exhibit synergistic effects in forming conductive networks [21, 22]. We explore such potential synergistic effects in polymer foams with hybrid CNT/GNP fillers.

In our simulations, both fillers in proper volume proportion and in total amount less than the percolation threshold are initially added to the solid cuboid. Once pores are progressively inserted into the cuboid, filler position relative to the pores is determined and, if necessary, fillers are moved out of the pores. A new challenge presented by CNT/GNP hybrid systems in foam simulations is the overlap of CNTs and GNPs. If a CNT or GNP, once moved out of the pore, intersects any other filler, we treat this as a contact and that CNT or GNP as part of the percolative network, just as we did for intersecting GNPs. In the algorithm, we preserve the filler dimensions and locations.

\section{Results and discussion}

\subsection{Percolation behavior in porous polymer/CNT nanocomposites}

In all simulations, we have employed CNTs with length $1.125 \mu \mathrm{m}$ and diameter $9.75 \mathrm{~nm}$, a cuboid edge length of $10 \mu \mathrm{m}$ and mean pore diameter of $2.25 \mu \mathrm{m}$.
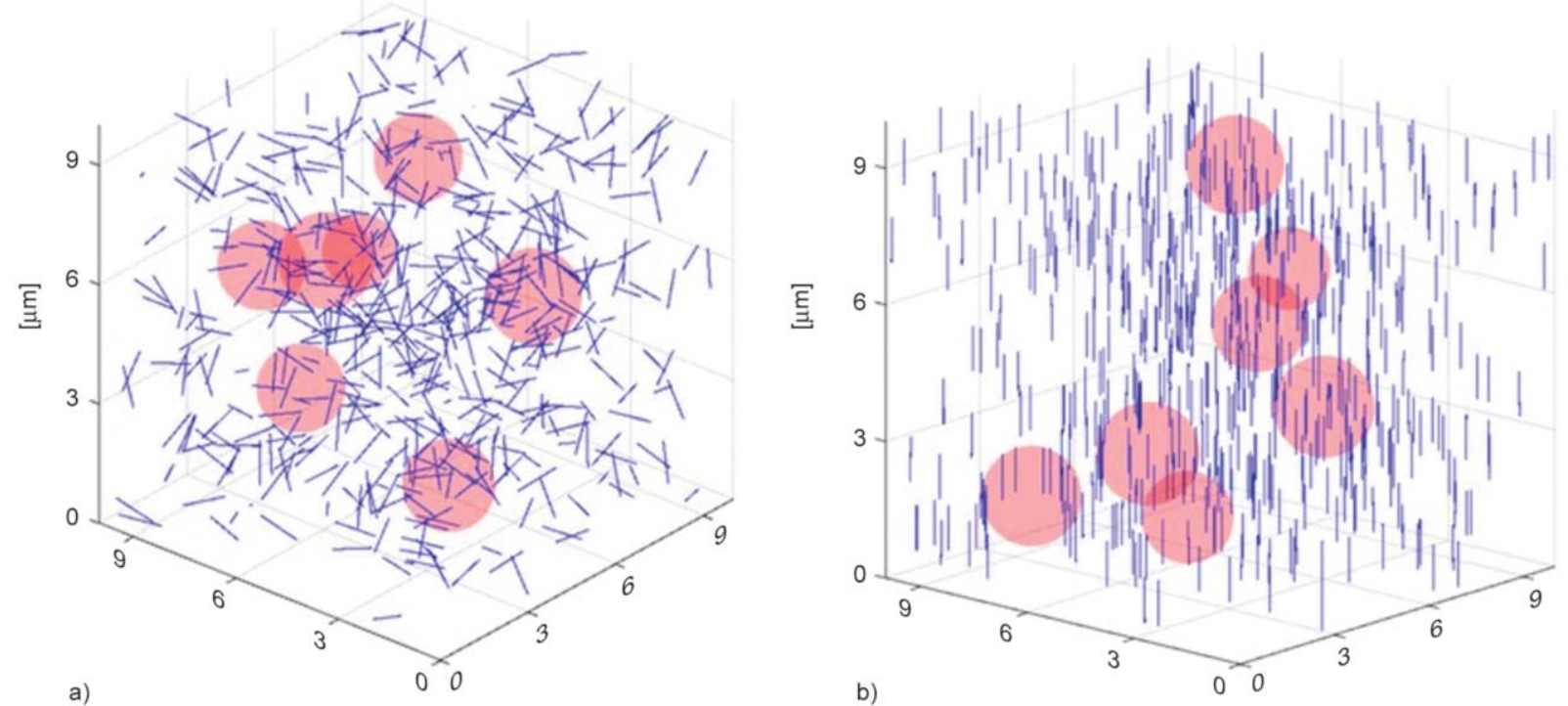

Figure 5. Visual representations of porous systems with (a) randomly aligned and (b) highly aligned CNT fillers. (Porosity level is $3-4 \%$ ). 
Figure 5 shows a visual representation of a porous system with randomly (Figure 5a) or highly aligned (Figure $5 b$ ) CNTs at porosity of 3-4\%.

Numerical results show that the percolation threshold of CNT porous systems significantly decreases with porosity level (Figure 6a). From a numerical point of view, a higher porosity leads to a higher number of pores and a decrease in average pore wall thickness ( $\sim 90 \%$ decrease is observed when increasing the porosity level from 10 to $40 \%$ ), indicating a greater probability of contact between fillers; therefore a lower number of fillers are required to form a conductive network. Numerical results were mostly in accordance with experimental observations [2]. The main rationale for the decrease in the measured percolation with the porosity level was related to a decrease in inter-particle distance. Specifically, the incorporation of pores in nanocomposites was responsible for a more dense filler network, with shorter path for electron transport, less electron scattering, and thus increased conductivity and reduced

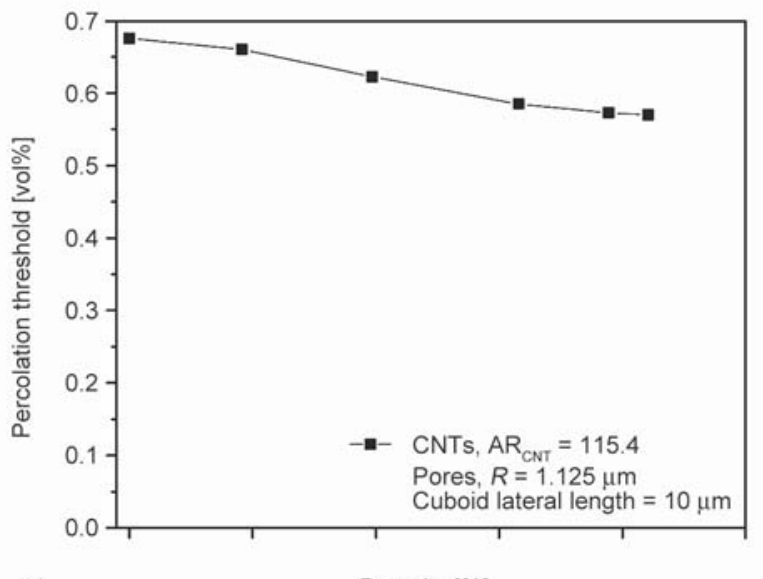

a)

Porosity [\%] percolation threshold [23]. Kim et al. reported experimental studies of polymer foams incorporating CNTs $[24,25]$. The authors observed an increase in electrical conductivity and decrease in percolation threshold with porosity when considering a fixed filler amount. On the other hand, other authors have described controversial results on the percolation behavior in porous systems with different porosity levels. In particular, concurrent mechanisms have been reported to play a role on the percolation behavior [26], specifically the biaxial stretching occurring during foaming was observed to cause the CNTs to slightly orient around the pores, while the pore-to-pore compression forced them to decrease their distance. This action increased the CNTs' interconnections and thereby increased the electrical conductivity and reduced the percolation value. However, as the degree of foaming was further increased beyond an optimum level $(>30$ $40 \%$ porosity, depending on the foam morphology and nanofiller type), excessive stretching resulted in concurrent filler orientation and increased distance

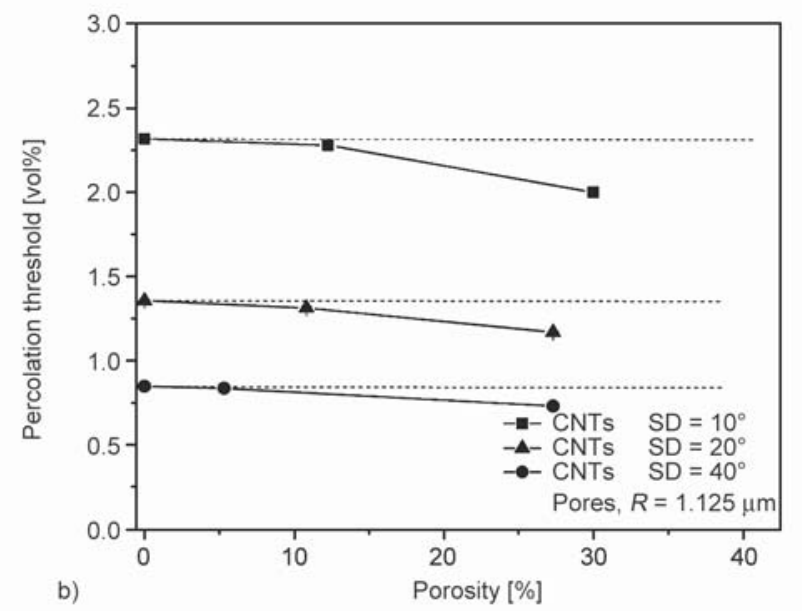

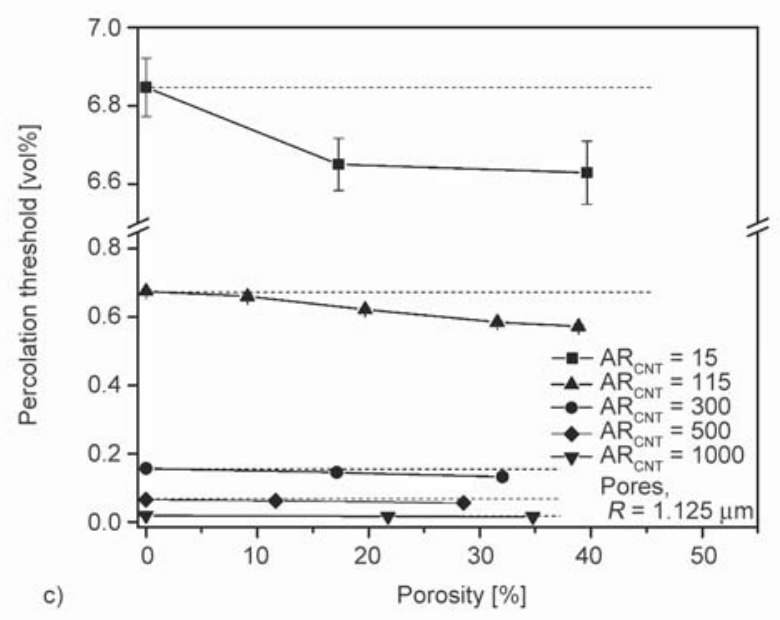

Figure 6. Dependency of the percolation threshold on (a) the porosity level, (b) CNT alignment, and (c) CNT aspect ratio. Dashed lines in $(\mathrm{b}-\mathrm{c})$ represent the percolation value in the case of bulk systems ( $0 \%$ porosity). Note that most of error bars are not shown because they are smaller than the symbol size. 
between fillers. Consequently, beyond this point, the percolation threshold was observed to increase proportionally with the porosity level. Similar experimental observations and interpretations have been reported by Tran and coworkers [27,28] and Zhi et al. [29] for porous nanocomposites incorporating multiwalled CNTs.

Some processing techniques using high aspect ratio fillers, including CNTs [25] and GNPs [30], can result in a morphology characterized by highly oriented fillers. Experimental observations have shown that electrical conductivity depends on filler alignment in solid nanocomposites. Specifically, it has been reported that neither a fully isotropic distribution nor a complete filler alignment are optimal to achieve the highest conductivity [30, 31]. However, Bao et al. [32] demonstrated that for very small volume fractions of CNTs, higher conductivities are achieved when the filler alignment is closer to isotropic. Specifically the percolation threshold in bulk systems will be higher for aligned fillers than for randomly oriented ones, primarily due to less effective filler interconnection for uniformly aligned fillers. Recent literature also reports the successful development of porous morphologies displaying preferentially aligned fillers by using manufacturing techniques such as microcellular injection molding; however little research on the electrical conductivity of such systems can be found [33].

We carried out numerical simulations to investigate the effect of filler orientation on percolation behavior in porous systems. We specify filler orientation with respect to one direction (in this case the $z$ direction) using the angle $\theta$, defined by Equation (10):

$z_{\mathrm{end}}-z_{\mathrm{start}}=L_{\mathrm{CNT}} \cdot \cos \theta$

where $z_{\text {end }}$ and $z_{\text {start }}$ are the $z$-coordinates of the CNT, $L_{\mathrm{CNT}}$ the length of the CNT, and the angle $\theta$ is described by a normal distribution centered on 0 (i.e. the default is for the CNTs to be uniformly oriented along the $z$ axis). The CNTs were aligned as they were inserted into the cuboid (prior to pore insertion) and their orientation was maintained as they were forced out of the pores. The standard deviation (SD) of the $\theta$ distribution was used to analyze the effects of misalignment on the percolation threshold. Numerical results indicate that higher percolation values are obtained when forcing the CNTs to orient completely (i.e. $\mathrm{SD}=0^{\circ}$ ) as the oriented CNTs are less effective in forming a percolative network around the pores. Figure $6 \mathrm{~b}$ presents the results for the effect of filler orientation on percolation threshold.

The effect of filler aspect ratio on percolation behavior in porous systems was analyzed by varying the smaller dimension of the CNTs (i.e. diameter), because such dimension is negligible with respect to the lateral length of the cuboid and the pore size [12]. As already observed in non-porous systems [12], higher aspect ratio fillers lead to lower percolation values (Figure 6c). Meanwhile, higher porosities lead to slightly lower percolation values, but there is no cross-dependence between changing aspect ratios and porosities. This is because the CNT rod-like geometry is more prone to confinement within the pore walls irrespective of aspect ratio.

\subsection{Percolation behavior in porous polymer/GNP nanocomposites}

In all simulations, we have employed GNPs with diameter $0.75 \mu \mathrm{m}$ and thickness $11.25 \mathrm{~nm}$, a cuboid edge length of $10 \mu \mathrm{m}$ and mean pore diameter of $2.25 \mu \mathrm{m}$. Figure 7 shows a visual representation of a porous system with randomly (Figure 7a) or highly aligned (Figure 7b) GNPs at a porosity level of 3-4\%. Numerical results show higher percolation thresholds for GNP systems with respect to CNT systems, as the randomly distributed 2D-GNPs are unable to form a percolation network as efficiently as the 1DCNTs [34]. Specifically, the 2D-GNPs seem less amenable than 1D-CNTs to adapt to the narrow walls between the cells, leading to a higher volume of fillers required to build the network. The percolation threshold of the GNP porous systems (Figure 8a) dramatically decreases with porosity, in agreement with some experimental studies [5], showing a greater dependence of percolation threshold on porosity by comparison with CNT systems ( $\sim 13$ and $\sim 55 \%$ decrease when increasing the porosity from 10 to $40 \%$ in CNT and GNP systems, respectively). This phenomenon occurs because of the difference in geometry between CNTs and GNPs. GNPs, due to larger volume and 2D shape, can 'reach out' further when confined inside the pore walls. That is, GNPs can more efficiently fill the space between the pores than CNTs. This causes a more dramatic effect of changing porosity on the percolation threshold for GNP systems.

We explored the effect of filler alignment on percolation threshold in porous systems with GNPs. In 

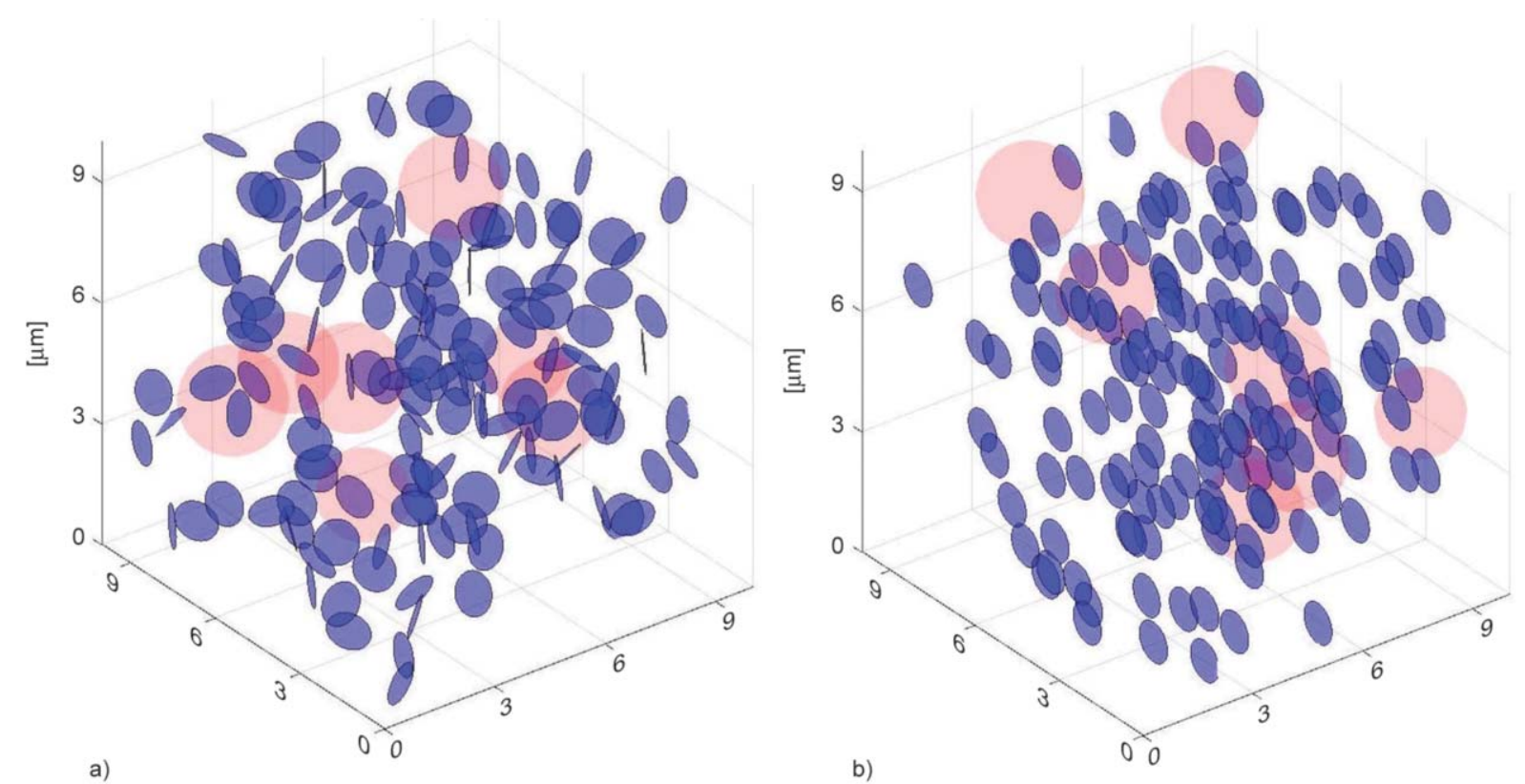

Figure 7. Visual representations of porous systems with (a) randomly aligned and (b) highly aligned GNP fillers. (Porosity level is 3-4\%).
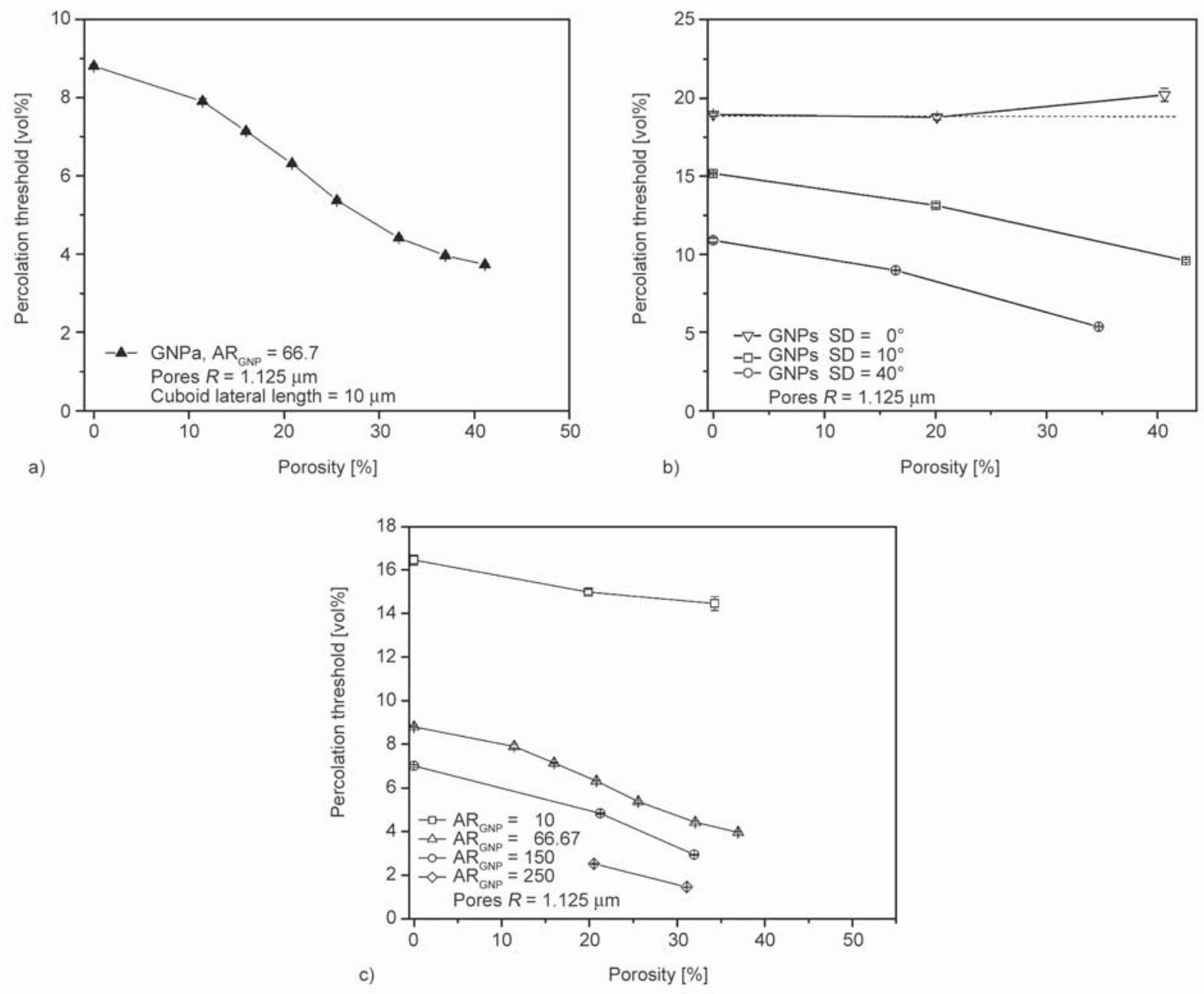

Figure 8. Dependency of the percolation threshold on (a) the porosity level, (b) GNP alignment, and (c) GNP aspect ratio. Dashed lines in (b) represent the percolation value in the case of bulk systems ( $0 \%$ porosity). Note that most of error bars are not shown because they are smaller than the symbol size. 
this case, filler alignment was examined by considering the angle $\theta$ between the vector normal to the platelet disk and the $x$-axis and defining a normal distribution on $\theta$ with mean zero and standard deviations between 0 and 40 degrees. Just as for CNT systems, the aligned GNPs were inserted into the cuboid prior to incorporating the pores and their orientation was maintained as they were moved out of the pores (Figure 7b).

Randomization of GNP alignment has a significant effect on the percolation threshold (Figure 8b). Specifically, a decrease of $55 \%$ in the percolation threshold is observed when considering a GNP system with $\sim 30 \%$ porosity and alignment $\theta=N(0,40)$ (i.e. $\theta$ normally distributed around $0^{\circ}$ with $\mathrm{SD}$ of $40^{\circ}$ ) with respect to the case $\theta=N(0,10)$ (i.e. $\theta$ normally distributed around $0^{\circ}$ with SD of $10^{\circ}$ ). Thus, a more randomized system (i.e. higher SD) can form networks using a lower initial volume of fillers. The limited available literature on the percolation behavior of aligned GNPs in non-porous systems [30] reports lower percolation thresholds for compression molded samples (acquiescent to a more randomized filler orientation) than for injection molded ones (conducive to more filler alignment).

The effect of filler aspect ratio on percolation behavior in porous systems with GNPs was simulated by varying only the dimension of the fillers that was negligible compared to the cuboid size (i.e. thickness) [12]. Due to excessive computational time requirements, simulations considering GNPs with AR $>100$ were not performed at low porosities.

GNPs with higher AR led to lower percolation values, while exhibiting also more efficient network formation at increased porosity levels. For example, for GNPs with $\mathrm{AR}=10$ at $30 \%$ porosity level, the percolation threshold is reached at approximately $90 \%$ of the filler amount required to form a network in a solid polymer. However, when using GNPs with $\mathrm{AR}=100$ at $30 \%$ porosity level, percolation is reached at only about $50 \%$ of the filler amount required to percolate in a solid (Figure 8c).

\subsection{Percolation in porous polymer/hybrid filler systems}

Several experimental studies report lower percolation thresholds for non-porous nanocomposites incorporating hybrid carbon nanofillers due to synergistic effects [21, 22, 35], however there are no reports on porous nanocomposites with hybrid carbon fillers. Therefore, the parametric study of the present model has the premise to shed some light on how one can maximize the benefits arising from using different fillers and tailor the porous morphology in order to minimize percolation threshold.

Numerical simulations were performed by inserting into the cuboid CNTs and GNPs in prescribed volume proportion with subsequent addition of pores until a percolative network was formed (Figure 9). We have used the same parameters as in the single filler simulations.

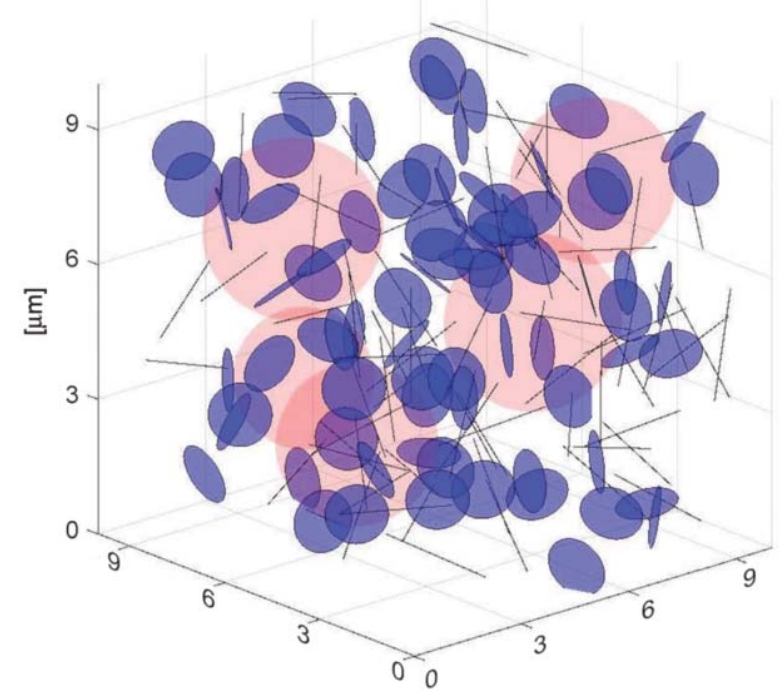

Figure 9. Visual representations of porous systems with hybrid $\mathrm{CNT}_{50} / \mathrm{GNP}_{50}$ filler. (Porosity level is $3-4 \%$ ).

Table 1. Percolation values and synergy of hybrid CNT/GNP systems at different levels of porosity

\begin{tabular}{|c|c|c|c|c|c|c|}
\hline \multirow[b]{2}{*}{ Nanofiller } & \multicolumn{2}{|c|}{ Porosity $=\mathbf{3 0} \%$} & \multicolumn{2}{|c|}{ Porosity $=35 \%$} & \multicolumn{2}{|c|}{ Porosity $=40 \%$} \\
\hline & $\begin{array}{c}\text { Percolation } \\
\text { [vol\%] }\end{array}$ & $\begin{array}{c}\text { Synergy } \\
{[\%]}\end{array}$ & $\begin{array}{c}\text { Percolation } \\
\text { [vol\%] }\end{array}$ & $\begin{array}{c}\text { Synergy } \\
{[\%]}\end{array}$ & $\begin{array}{c}\text { Percolation } \\
\text { [vol\%] }\end{array}$ & $\begin{array}{c}\text { Synergy } \\
{[\%]}\end{array}$ \\
\hline CNT & $0.590 \pm 0.003$ & 0.0 & $0.579 \pm 0.003$ & 0.0 & $0.572 \pm 0.003$ & 0.0 \\
\hline $\mathrm{CNT}_{70} / \mathrm{GNP}_{30}$ & $0.814 \pm 0.004$ & 55.5 & $0.797 \pm 0.004$ & 51.6 & $0.792 \pm 0.003$ & 48.6 \\
\hline $\mathrm{CNT}_{50} / \mathrm{GNP}_{50}$ & $1.086 \pm 0.007$ & 59.1 & $1.062 \pm 0.003$ & 55.0 & $1.050 \pm 0.007$ & 51.9 \\
\hline $\mathrm{CNT}_{30} / \mathrm{GNP}_{70}$ & $1.641 \pm 0.008$ & 52.8 & $1.597 \pm 0.011$ & 48.1 & $1.585 \pm 0.018$ & 44.0 \\
\hline GNP & $4.718 \pm 0.036$ & 0.0 & $4.145 \pm 0.027$ & 0.0 & $3.796 \pm 0.020$ & 0.0 \\
\hline
\end{tabular}

Synergy values are expressed with respect to the relative amount by which the percolation threshold varies from the prediction of the ROM. 


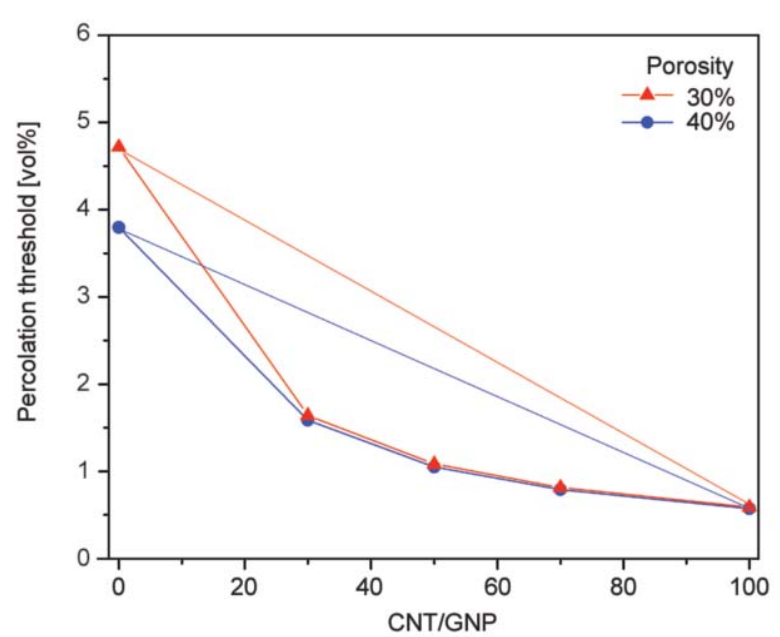

Figure 10. Dependency of the percolation threshold on the CNT/GNP ratio in hybrid systems. Solid lines represent predictions according to the ROM

The numerical simulation results are shown in Table 1. As expected from the single-filler simulations, systems with higher proportions of GNPs showed greater dependency of the percolation threshold on porosity, while systems with more CNTs were less sensitive to porosity levels.

Furthermore, the hybrid systems exhibited significantly lower percolation values in porous systems when compared to the predictions of the rule of mixture (ROM), indicating significant synergistic effects (Figure 10). Here synergy was determined as the percentage difference of the measured value with respect to the value given from the rule of mixture (i.e. linear relationship).

\section{Conclusions}

The electrical percolation behavior of porous systems incorporating carbon 1D- and 2D-fillers was numerically investigated assuming perfectly random filler distribution and realistically modeling 'foaming' by displacing fillers. The model was able to capture successfully experimental trends that show increased conductivities (and lower percolation thresholds) in porous systems compared to solid ones. Sensitivity analysis found that the electrical percolation behavior of porous polymer systems incorporating 1D- and 2D-nanofillers was significantly affected by (i) porosity level, (ii) type of filler, (iii) filler alignment, and (iv) filler AR. The type of filler and its AR played the most important roles in determining percolation threshold.
Numerical results revealed a noticeable difference between CNT and GNP porous systems, indicating that: (i) lower percolation values and reduced dependency on the porosity level are achieved when using 1D-CNTs in comparison with 2D-GNPs, (ii) CNTs are more efficient in forming a percolative network than GNPs, especially when using high-AR fillers, and (iii) hybrid systems incorporating CNTs and GNPs exhibit significant synergistic effects.

\section{Acknowledgements}

The authors gratefully thank Drs. Edward Conjura and Michael Ochs (Department of Mathematics and Statistics, The College of New Jersey - NJ) for many useful discussions and suggestions on the numerical code and simulation procedure. We also acknowledge the National Science Foundation for partial financial support of our research through grant PIRE-1243313.

\section{References}

[1] Lee L. J., Zeng C., Cao X., Han X., Shen J., Xu G.: Polymer nanocomposite foams. Composites Science and Technology, 65, 2344-2363 (2005).

https://doi.org/10.1016/j.compscitech.2005.06.016

[2] Xu X-B., Li Z-M., Shi L., Bian X-C., Xiang Z-D.: Ultralight conductive carbon-nanotube-polymer composite. Small, 3, 408-411 (2007).

https://doi.org/10.1002/smll.200600348

[3] Baltopoulos B., Athanasopoulos N., Matzakou M., Vavouliotis A., Kostopoulos V.: Polymer foams with tailored electrical conductivity using multiwall carbon nanotubes. SPE Plastics Research Online, p.4 (2012). https://doi.org/10.2417/spepro.004333

[4] Yang Y., Gupta M. C., Dudley K. L., Lawrence R. W.: Conductive carbon nanofiber-polymer foam structures. Advanced Materials, 17, 1999-2003 (2005). https://doi.org/10.1002/adma.200500615

[5] Gedler G., Antunes M., Velasco J. I.: Enhanced electrical conductivity in graphene-filled polycarbonate nanocomposites by microcellular foaming with $\mathrm{sc}-\mathrm{CO}_{2}$. Journal of Adhesion Science and Technology, 30, 10171029 (2016).

http://dx.doi.org/10.1080/01694243.2015.1137700

[6] Kostopoulos V., Vavouliotis A., Karapappas P., Tsotra P., Paipetis A.: Damage monitoring of carbon fiber reinforced laminates using resistance measurements. Improving sensitivity using carbon nanotube doped epoxy matrix system. Journal of Intelligent Material Systems and Structures, 20, 1025-1034 (2009). https://doi.org/10.1177/1045389X08099993

[7] Badamshina E., Estrin Y., Gafurova M.: Nanocomposites based on polyurethanes and carbon nanoparticles: Preparation, properties and application. Journal of Materials Chemistry A, 1, 6509-6529 (2013). https://doi.org/10.1039/c3ta10204a 
[8] Potts J. R., Dreyer D. R., Bielawski C. W., Ruoff R. S.: Graphene-based polymer nanocomposites. Polymer, 52, 5-25 (2011).

https://doi.org/10.1016/j.polymer.2010.11.042

[9] Cai D., Song M.: Recent advance in functionalized graphene/polymer nanocomposites. Journal of Materials Chemistry, 20, 7906-7915 (2010).

https://doi.org/10.1039/c0jm00530d

[10] Stevens D. R., Downen L. N., Clarke L. I.: Percolation in nanocomposites with complex geometries: Experimental and Monte Carlo simulation studies. Physical Review B, 78, 235425/1-235425/5 (2008).

https://doi.org/10.1103/PhysRevB.78.235425

[11] Behbahani A. F., Motlagh G. H., Ziaee M., Nikravan G.: Electrical percolation behavior of carbon fiber and carbon nanotube polymer composite foams: Experimental and computational investigations. Journal of Applied Polymer Science, 132, 42685/1-42685/12 (2015). https://doi.org/10.1002/APP.42685

[12] Maxian O., Pedrazzoli D., Manas-Zloczower I.: Modeling the electrical percolation behavior of hybrid nanocomposites based on carbon nanotubes and graphene nanoplatelets. Materials Research Express, 2, 095013/1095013/15 (2015).

https://doi.org/10.1088/2053-1591/2/9/095013

[13] Oskouyi A., Sundararaj U., Mertiny P.: Tunneling conductivity and piezoresistivity of composites containing randomly dispersed conductive nano-platelets. Materials, 7, 2501-2521 (2014). https://doi.org/10.3390/ma7042501

[14] Li J., Kim J-K.: Percolation threshold of conducting polymer composites containing 3D randomly distributed graphite nanoplatelets. Composites Science and Technology, 67, 2114-2120 (2007).

https://doi.org/10.1016/j.compscitech.2006.11.010

[15] Hu N., Masuda Z., Yan C., Yamamoto G., Fukunaga H., Hashida T.: The electrical properties of polymer nanocomposites with carbon nanotube fillers. Nanotechnology, 19, 215701/1-215701/10 (2008). https://doi.org/10.1088/0957-4484/19/21/215701

[16] Ambrosetti G., Grimaldi C., Balberg I., Maeder T., Danani A., Ryser P.: Solution of the tunneling-percolation problem in the nanocomposite regime. Physical Review B, 81, 155434/1-155434/23 (2010). https://doi.org/10.1103/PhysRevB.81.155434

[17] Ramôa S. D. A. S., Barra G. M. O., Oliveira R. V. B., de Oliveira M. G., Cossa M., Soares B. G.: Electrical, rheological and electromagnetic interference shielding properties of thermoplastic polyurethane/carbon nanotube composites. Polymer International, 62, 1477-1484 (2013).

https://doi.org/10.1002/pi.4446

[18] Almohamad H. A., Selim S. Z.: An algorithm for computing the distance between two circular disks. Applied Mathematical Modelling, 27, 115-124 (2003). https://doi.org/10.1016/S0307-904X(02)00080-X
[19] Chung D. D. L.: Exfoliation of graphite. Journal of Materials Science, 22, 4190-4198 (1987).

https://doi.org/10.1007/BF01132008

[20] Kalaitzidou K., Fukushima H., Drzal L. T.: Processing, properties and structure of exfoliated graphite nanoplatelet-polypropylene nanocomposites. in 'AIChE Annual Meeting, Conference Proceedings, Cincinnati, USA' 13647-13665 (2007).

[21] Yue L., Pircheraghi G., Monemian S. A., Manas-Zloczower I.: Epoxy composites with carbon nanotubes and graphene nanoplatelets - Dispersion and synergy effects. Carbon, 78, 268-278 (2014). https://doi.org/10.1016/j.carbon.2014.07.003

[22] Safdari M., Al-Haik M.: Electrical conductivity of synergistically hybridized nanocomposites based on graphite nanoplatelets and carbon nanotubes. Nanotechnology, 23, 405202/1-405202/10 (2012). https://doi.org/10.1088/0957-4484/23/40/405202

[23] Rizvi R., Naguib H.: Porosity and composition dependence on electrical and piezoresistive properties of thermoplastic polyurethane nanocomposites. Journal of Materials Research, 28, 2415-2425 (2013).

https://doi.org/10.1557/jmr.2013.218

[24] Kim Y.: Polymer nanocomposite foams: Fabrication, characterization, and modeling. PhD thesis, The University of Texas at Austin (2012).

[25] Kim Y-H., Li W.: Multifunctional polyetherimide nanocomposite foam. Journal of Cellular Plastics, 49, 131145 (2013). https://doi.org/10.1177/0021955X13477434

[26] Ameli A., Nofar M., Park C. B., Pötschke P., Rizvi G.: Polypropylene/carbon nanotube nano/microcellular structures with high dielectric permittivity, low dielectric loss, and low percolation threshold. Carbon, 71, 206-217 (2014).

https://doi.org/10.1016/j.carbon.2014.01.031

[27] Tran M-P., Detrembleur C., Alexandre M., Jerome C., Thomassin J-M.: The influence of foam morphology of multi-walled carbon nanotubes/poly(methyl methacrylate) nanocomposites on electrical conductivity. Polymer, 54, 3261-3270 (2013).

https://doi.org/10.1016/j.polymer.2013.03.053

[28] Tran M-P., Thomassin J-M., Alexandre M., Jerome C., Huynen I., Detrembleur C.: Nanocomposite foams of polypropylene and carbon nanotubes: Preparation, characterization, and evaluation of their performance as EMI absorbers. Macromolecular Chemistry Physics, 216, 1302-1312 (2015). https://doi.org/10.1002/macp.201500031

[29] Zhi X., Zhang H-B., Liao Y-F., Hu Q-H., Gui C-X., Yu Z-Z.: Electrically conductive polycarbonate/carbon nanotube composites toughened with micron-scale voids. Carbon, 82, 195-204 (2015).

https://doi.org/10.1016/j.carbon.2014.10.062 
[30] Kalaitzidou K., Fukushima H., Drzal L. T.: A new compounding method for exfoliated graphite-polypropylene nanocomposites with enhanced flexural properties and lower percolation threshold. Composites Science and Technology, 67, 2045-2051 (2007).

https://doi.org/10.1016/j.compscitech.2006.11.014

[31] Du F., Fischer J., Winey J. K.: Effect of nanotube alignment on percolation conductivity in carbon nanotube/ polymer composites. Physical Review B, 72, 121404/1121404/4 (2005).

https://doi.org/10.1103/PhysRevB.72.121404

[32] Bao W. S., Meguid S. A., Zhu Z. H., Meguid M. J.: Modeling electrical conductivities of nanocomposites with aligned carbon nanotubes. Nanotechnology, 22, 485704/1-485704/8 (2011).

https://doi.org/10.1088/0957-4484/22/48/485704
[33] Kharbas H., Nelson P., Yuan M., Gong S., Turng L-S.: Effects of nano-fillers and process conditions on the microstructure and mechanical properties of microcellular injection molded polyamide nanocomposites. Polymer Composites, 24, 655-671 (2003).

https://doi.org/10.1002/pc. 10060

[34] Safdari M., Al-Haik M. S.: Synergistic electrical and thermal transport properties of hybrid polymeric nanocomposites based on carbon nanotubes and graphite nanoplatelets. Carbon, 64, 111-121 (2013).

https://doi.org/10.1016/j.carbon.2013.07.042

[35] Song P., Liu L., Fu S., Yu Y., Jin C., Wu Q., Zhang Y., Li Q.: Striking multiple synergies created by combining reduced graphene oxides and carbon nanotubes for polymer nanocomposites. Nanotechnology, 24, 125704/1125704/8 (2013).

https://doi.org/10.1088/0957-4484/24/12/125704 